\title{
Adjuvant therapy of pancreatic carcinoma: the experience of Policlinico Umberto I, Università "Sapienza" Rome
}

This article was published in the following Dove Press journal:

Gastrointestinal Cancer:Targets and Therapy

9 August 2013

Number of times this article has been viewed

\section{Francesca De Felice \\ Daniela Musio \\ Raffaele Cassese \\ Daria Masi \\ Vincenzo Tombolini}

Dipartimento di Scienze Radiologiche Oncologiche e Anatomo-Patologiche, Cattedra di Radioterapia, Università degli Studi di Roma "Sapienza", Rome, Italy
Correspondence: Francesca De Felice Dipartimento di Scienze Radiologiche Oncologiche e Anatomo-Patologiche, Cattedra di Radioterapia, Università degli Studi di Roma "Sapienza", Viale Regina Elena I55, 00I6I Rome, Italy

Tel +390649973404

Fax +3906 499734II

Email brency7@hotmail.it
Background: Pancreatic cancer represents an unsolved oncological problem: only $10 \%-20 \%$ of patients have resectable disease at diagnosis. We describe our adjuvant treatment approach and our results of subsequent multidisciplinary care of resected pancreatic cancer patients.

Materials and methods: Between January 2007 and May 2012, 17 patients with resected pancreatic cancer underwent adjuvant radiochemotherapy. Radiotherapy was delivered with a three-dimensional-conformational multiple field technique at a total dose of 50.4-54.0 Gy. Chemotherapy consisted of gemcitabine $\left(200 \mathrm{mg} / \mathrm{m}^{2} /\right.$ week $)$ or fluorouracil $\left(200 \mathrm{mg} / \mathrm{m}^{2} /\right.$ day $)$.

Results: 16 patients completed programmed treatment; one patient suspended planned adjuvant treatment because of hematological toxicity grade 3 . He was the only case of toxicity grade 3 . At a median follow-up of 17.7 months, nine patients are still disease-free survivors. The 2-year survival was $30 \%$.

Discussion: Our experience in resected pancreatic cancer patients treated with a radiochemotherapy regimen highlights the survival and toxicity profile benefits associated with concomitant treatment.

Keywords: pancreatic cancer, adjuvant treatment, pancreas

\section{Introduction}

Pancreatic cancer represents a difficult and unsolved oncological problem. Most patients with pancreatic cancer present with advanced disease that is not eligible for curative resection; only $10 \%-20 \%$ of patients have resectable disease at diagnosis. The prognosis is poor, with a 5-year survival rate of $6 \% .{ }^{1}$ Even so, surgical resection, as a part of a multimodality treatment approach, remains the only potentially curative treatment strategy. The addition of nonsurgical treatment, such as adjuvant therapy, has been employed to achieve at least a modest improvement in survival. Despite multiple trials, the definitive role for adjuvant therapy has not been established, and it represents one of the most debated topics in oncology in the modern age. ${ }^{2}$ While the role of adjuvant radiochemotherapy is still controversial, extensive evidence exists for the use of adjuvant chemotherapy. ${ }^{3-9}$

This paper describes the adjuvant treatment approach in our department and the results of subsequent multidisciplinary care of resected pancreatic cancer patients.

\section{Materials and methods}

A total of 17 patients (five female, 12 male) with histologically confirmed resectable adenocarcinoma of the pancreas, treated between January 2007 and May 2012, were included in this study. Median patient age was 63.3 years (range 41-80 years). 
The clinical presentation resulted in jaundice in nine patients, pain in six patients, and weight loss in two patients. According to the American Joint Committee on Cancer, ${ }^{10}$ tumor size, lymph nodes affected, metastases staging system 2010 for pancreatic cancer, ten patients showed pathological lymph nodes. All patients underwent surgery before radiochemotherapy: ten patients were pathologically staged as stage IIB, three patients as stage IIA and four patients as stage I. Only one patient had a positive margin. The characteristics of patients are listed in Table 1.

All patients underwent radiochemotherapy. A computed tomography (CT) scan was performed with the patient supine in the treatment position: arms folded across the chest and legs restrained with ankle supports. Slices $5 \mathrm{~mm}$ thick were obtained from the level of thoracic vertebra 11 for appropriate margins on the celiac vessel, to the lower border of lumbar vertebra 3 to cover the kidneys. Midline and lateral tattoos were used with laser lights to align the patient and prevent lateral rotation. CT data were transferred to the Treatment Planning Software (Pinnacle ${ }^{\circledR}$; Philips Radiation Oncology Systems, Fitchburg, WI, USA) to ascertain target volume definition and dose solutions. The planning target volume 1 (PTV1) included tumor bed and regional node (pancreaticoduodenal, porta hepatic, celiac, and suprapancreatic lymph nodes; superior mesenteric lymph nodes were included in head of pancreas lesions; splenic hilum nodes were included with pancreatic body or tail lesions). The planning target volume 2 (PTV2) included tumor bed with a $1.5 \mathrm{~cm}$, three-dimensional margin, including any areas of positive margin. Patients were set up for radiotherapy daily. Portal images were taken weekly and compared with DRRs (Digital Reconstructed Radiographs) from the planning CT scan.

Table I Characteristics of patients

\begin{tabular}{lll}
\hline Characteristics & $\mathbf{n}$ & $\%$ \\
\hline Sex & 12 & 71 \\
$\quad$ Male & 5 & 29 \\
$\quad$ Female & & \\
Age & & \\
$\quad$ Mean (63.3 years) & & \\
$\quad$ Range (4I-80 years) & 4 & 23 \\
Stage & 3 & 18 \\
I & 10 & 59 \\
IIA & & 100 \\
IIB & 17 & \\
Histology & & 59 \\
$\quad$ Adenocarcinoma & 10 & 41 \\
Adjuvant treatment & 7 & \\
RT-gemcitabine & RT-fluorouracil &
\end{tabular}

Abbreviations: \%, percent of total patients in the study; $n$, number of patients; $\mathrm{RT}$, radiation therapy.
Radiation therapy was delivered with a three-dimensionalconformational multiple field technique at a dose of $45 \mathrm{~Gy}$ (in 25 daily fractions of 1.8 Gy given in 5 weeks) to the PTV1, plus a 5.4-9.0 Gy (in 3-5 daily fractions of $1.8 \mathrm{~Gy}$ ) to the field boost - PTV2 - with 6-15 MV energy photons.

Patients underwent the insertion of a central venous access catheter (port-a-cath) for chemotherapy delivery. Chemotherapy treatment was left to the oncologist's discretion and consisted of gemcitabine $200 \mathrm{mg} / \mathrm{m}^{2}$ by bolus intravenous injection on the first day of each week of radiotherapy or fluorouracil (5-FU) $200 \mathrm{mg} / \mathrm{m}^{2}$ five daily continuous infusions. Gemcitabine was administered in ten patients and 5-FU in seven patients. In all patients, dexamethasone $(8 \mathrm{mg})$ and ondansetron $(8 \mathrm{mg}$ ) were administered before the adjuvant treatment. Figure 1 shows the adjuvant treatment protocol.

After adjuvant treatment, all patients underwent careful physical examination and total body CT scan at 2-month intervals to evaluate for disease recurrence/progression. Overall survival and disease-free survival were considered in months from the end of the adjuvant treatment.

\section{Results}

All patients received concomitant radiochemotherapy: 16 patients completed programmed treatment, and one patient suspended definitively planned adjuvant concomitant treatment because of hematological toxicity grade 3 after 16 days. The patient started health care.

Toxicity was evaluated according to National Cancer Institute - Common Terminology Criteria, version 3.0. ${ }^{11}$ Excluding the previous patient for whom health care was activated, there was no hematological toxicity; gastrointestinal toxicity adverse events were $\leq$ grade 2 and they were registered in five patients only.

No patients were lost to follow-up. At an average followup of 17.7 months and a median survival of 13 months

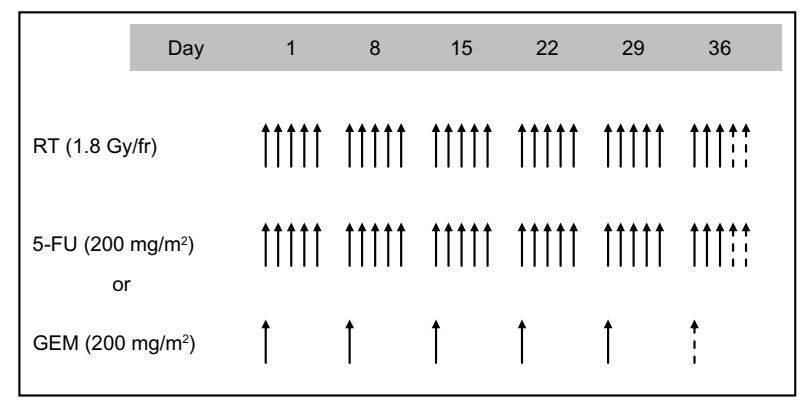

Figure I Adjuvant treatment protocol.

Notes: Patients received 50.4 Gy (solid arrows) or 54.0 Gy (dashed arrows) of radiation therapy (RT) and 28 days (solid arrows) or 30 days (dashed arrows) of fluorouracil (5-FU) or five (solid arrows) or six (dashed arrow) weekly cycles of gemcitabine (GEM) dependent on oncologist's discretion. 
(range 1-56), nine patients are still disease-free survivors; one patient is still alive, but he presented liver metastases 17 months after the end of adjuvant treatment. The 2-year survival was $30 \%$. In the full analysis of the population, five patients developed distant metastases and three patients showed local progression. All the long-term survivors were negative-node at diagnosis. In a subgroup analysis, no differences in terms of toxicity were detected between gemcitabine and 5-FU chemotherapy. Moreover, the gemcitabine regimen provided the same local control with no difference in overall survival compared to 5-FU radiochemotherapy.

\section{Discussion}

Over the years, chemotherapy and radiotherapy have emerged as valuable adjuncts to the management of several cancers. For breast cancer, glioblastoma multiforme, and colorectal cancer, randomized controlled trials have proven the survival benefits in resectable cases, therefore standard adjuvant therapy is universally accepted. ${ }^{12-14}$ For head and neck cancer and gastric cancer, adjuvant radiochemotherapy is considered a standard treatment in patients with resectable and advanced disease, despite the clash of opinions regarding which drugs could be associated with radiation therapy. ${ }^{15,16}$ For resectable pancreatic cancer, however, no globally accepted standard adjuvant therapy has yet been established. ${ }^{3}$ Few controversies are associated with adjuvant therapy for pancreatic cancer. Primarily, it is difficult to detect pancreatic cancer at the resectable stage, and consequently, the small number of cases makes it difficult to conduct a randomized controlled trial. Moreover, the management of resected pancreatic cancer remains to be clarified because there is no global consensus between Europe and the US regarding adjuvant treatment, even though the significant rates of local and distant recurrence imply that radiochemotherapy should be most appropriate. In Europe, it is common practice for patients to receive adjuvant chemotherapy, while most US physicians support the validity of adjuvant radiochemotherapy. ${ }^{2,3}$ There are several randomized controlled trials that support the use of adjuvant chemotherapy in resected pancreatic cancer: the European Study Group for Pancreatic Cancer (ESPAC)-1 trial, ${ }^{4}$ the ESPAC-3 trial, ${ }^{5}$ and the Charité Onkologie Clinical Studies in GI Cancer-001study. ${ }^{6}$ These trials demonstrated a survival benefit for adjuvant chemotherapy ${ }^{4,5}$ and a more tolerable toxicity profile with gemcitabine. ${ }^{6}$ The role of adjuvant radiochemotherapy has been investigated with as much interest in the US (Gastrointestinal Tumor Study Group trial and Radiation Therapy Oncology Group 97-04 trial) and in Europe (European Organisation for Research and Treatment of Cancer trial and ESPAC-1 trial). ${ }^{4,7-9}$ However, it remains controversial because every one of the randomized trials had many criticisms, such as potential bias, suboptimal radiation dose, slow accrual, and confusing study design. The Radiation Therapy Oncology Group 97-04 trial did not test the question of radiochemotherapy versus chemotherapy, such as the Charité Onkologie Clinical Studies in GI Cancer-001 trial; the European Organisation for Research and Treatment of Cancer trial was criticized because it was statistically underpowered; the ESPAC-1 trial was criticized because of the nihilistic conclusion that chemoradiotherapy had no role in the adjuvant therapy of resected pancreatic cancer. ${ }^{17}$ Even so, adjuvant radiochemotherapy has been accepted as appropriate adjuvant therapy in the US, since the Gastrointestinal Tumor Study Group study suggested a beneficial role for combined radiochemotherapy, despite the relatively small number of patients enrolled (43 patients) and the low radiation total dose delivered (40 Gy). ${ }^{4}$ Additional evidence for the benefit of adjuvant radiochemotherapy for resected pancreatic cancer has been provided in reports of single-institution experiences. ${ }^{18-21}$ The Johns Hopkins-Mayo Clinic collaborative study, ${ }^{18}$ the largest of these series, showed an improvement in median survival (20 months versus 14 months) and a better 2-year overall survival in patients who received adjuvant radiochemotherapy compared to patients who were not treated ( $45 \%$ versus $31 \%$, respectively). Like the previous study, the Mayo Clinic experience demonstrated that overall survival is better in patients who received adjuvant radiochemotherapy, ${ }^{19}$ and analysis of the Surveillance, Epidemiology, and End Results registry data demonstrated that adjuvant radiotherapy is associated with improved survival. ${ }^{20,21}$ Our retrospective data are concordant with these prior reports: compared to data in the literature, we revealed a survival benefit for adjuvant radiochemotherapy, and according to Yovino et $\mathrm{al},{ }^{22}$ the predominant failure pattern was distant metastases. Moreover, the ascertainment of a higher survival in patients with negative nodes at diagnosis confirmed that in order to increase survival, it is essential to detect pancreatic carcinoma at an early stage. Because combined adjuvant therapy is criticized as an inducer of intense, acute toxicity, ${ }^{3}$ it is important that radiation oncologists understand the postsurgical anatomy after pancreaticoduodenectomy to establish the effective treatment volume, while limiting dose exposure to normal tissue. In terms of toxicity, our protocol was well-tolerated, with only one case of hematological toxicity grade 3 . The limitation of our trial is its small number of patients, which does not allow for the solid comparison between sex and the two different chemotherapeutic regimens 
adopted (gemcitabine versus 5-FU). A key observation was that compliance to concomitant treatment was high and successful disease control was achieved. In comparison with other single-institution experiences, ${ }^{18-21}$ a similar survival and a better toxicity profile were observed. It is necessary to increase the sample size to a larger cohort of patients to confirm that high dose of radiation therapy and a relatively low dose of chemotherapy could be essential for survival and toxicity profile benefits. Apart from personalized adjuvant therapy, ${ }^{23,24}$ it would seem unreasonable to improve survival by giving up radiochemotherapy, because, despite the small cohort, our results confirm that concomitant treatment shows substantial local control. Distant metastasis remains a significant clinical problem.

Briefly, pancreatic cancer is still one of the most aggressive cancers. The addition of adjuvant radiotherapy and/or chemotherapy has realized a modest improvement in survival. Despite the well-supported role of chemotherapy, the role of radiation therapy continues to be investigated. Our experience with resected pancreatic cancer patients treated with a radiochemotherapy regimen highlights the survival and toxicity profile benefits associated with concomitant treatment.

\section{Disclosure}

The authors report no conflicts of interest in this work.

\section{References}

1. Siegel R, Naishadham D, Jemal A, Cancer statistics, 2012. CA Cancer J Clin. 2012;62(1):10-29.

2. Sharma C, Horowitz D, Chabot J, Saif MW. Adjuvant therapy of pancreatic cancer. Highlights from the "2011 ASCO Annual Meeting". Chicago, IL, USA; June 3-7, 2011. JOP. 2011;12(4):343-346.

3. Ueno H, Kosuge T. Adjuvant treatments for resectable pancreatic cancer. J Hepatobiliary Pancreat Surg. 2008;15(5):468-472.

4. Neoptolemos JP, Stocken DD, Friess H, et al; European Study Group for Pancreatic Cancer. A randomized trial of chemoradiotherapy and chemotherapy after resection of pancreatic cancer. $N$ Engl $\mathrm{J} \mathrm{Med}$. 2004;350(12):1200-1210.

5. Neoptolemos JP, Stocken DD, Bassi C et al; European Study Group for Pancreatic Cancer. Adjuvant chemotherapy with fluorouracil plus folinic acid vs gemcitabine following pancreatic cancer resection: a randomized controlled trial. JAMA. 2010;304(10):1073-1081.

6. Oettle H. Adjuvant chemotherapy with gemcitabine vs observation in patients undergoing curative-intent resection of pancreatic cancer: a randomized controlled trial. JAMA. 2007;297(3):267-277.

7. Klinkenbijl JH, Jeekel J, Sahmoud T, et al. Adjuvant radiotherapy and 5 -fluorouracil after curative resection of cancer of the pancreas and periampullary region: phase III trial of the EORTC gastrointestinal tract cancer cooperative group. Ann Surg. 1999;230(6):776-782.

8. Kalser MH, Ellenberg SS. Pancreatic cancer. Adjuvant combined radiation and chemotherapy following curative resection. Arch Surg. 1985;120(8):899-903.

9. Regine WF, Winter KA, Abrams RA, et al. Fluorouracil vs gemcitabine chemotherapy before and after fluorouracil-based chemoradiation following resection of pancreatic adenocarcinoma: a randomized controlled trial. JAMA. 2008;299(9):1019-1026. Erratum in: JAMA. 2008;299(16):1902.
10. AJCC: Edge S, Byrd D, Compton C, et al., eds. AJCC cancer staging manual, 7th ed. New York, NY: Springer Verlag, 2009.

11. http://ctep.cancer.gov [homepage on the Internet]. Common Terminology Criteria for Adverse Events, Version 3.0. Cancer Therapy Evaluation Program. National Cancer Institute; 2006 [updated August 9, 2006]. Available from: http://ctep.cancer.gov/protocolDevelopment/ electronic_applications/docs/ctcaev3.pdf.

12. Bellon JR, Harris EE, Arthur DW, et al; American College of Radiology. ACR Appropriateness Criteria ${ }^{\circledR}$ conservative surgery and radiation - stage I and II breast carcinoma: expert panel on radiation oncology: breast. Breast J. 2011;17(5):448-455.

13. Stupp R, Hegi ME, Mason WP, et al; European Organisation for Research and Treatment of Cancer Brain Tumour and Radiation Oncology Groups; National Cancer Institute of Canada Clinical Trials Group. Effects of radiotherapy with concomitant and adjuvant temozolomide versus radiotherapy alone on survival in glioblastoma in a randomised phase III study: 5-year analysis of the EORTC-NCIC trial. Lancet Oncol. 2009;10(5):459-466.

14. Metzger U. Adjuvant therapy for colon and rectal cancer. NIH Consensus Development Conference. Eur J Cancer. 1990;26(6):753-755.

15. Cooper JS, Pajak TF, Forastiere AA, et al; Radiation Therapy Oncology Group 9501/Intergroup. Postoperative concurrent radiotherapy and chemotherapy for high-risk squamous-cell carcinoma of the head and neck. N Engl J Med. 2004;350(19):1937-1944.

16. Ychou M, Gory-Delabaere G, Blanc P, et al; Fédération National des Centres de Lutte Contre le Cancer; Fédération Hospitalière de France; Fédération Française de Cancérologie. [Clinical practice guidelines: 2004 Standards, Options and Recommendations for the management of patient with adenocarcinoma of the stomach-radiotherapy]. Cancer Radiother. 2004;8(5):322-335. French.

17. Koshy MC, Landry JC, Cavanaugh SX, et al. A challenge to the therapeutic nihilism of ESPAC-1. Int J Radiat Oncol Biol Phys. 2005;61(4):965-966.

18. Hsu CC, Herman JM, Corsini MM, et al. Adjuvant chemoradiation for pancreatic adenocarcinoma: the Johns Hopkins Hospital-Mayo Clinic collaborative study. Ann Surg Oncol. 2010;17(4):981-990.

19. Corsini MM, Miller RC, Haddock MG, et al. Adjuvant radiotherapy and chemotherapy for pancreatic carcinoma: the Mayo Clinic experience (1975-2005). J Clin Oncol. 2008;26(21):3511-3516.

20. Hazard L, Tward JD, Szabo A, Shrieve DC. Radiation therapy is associated with improved survival in patients with pancreatic adenocarcinoma: results of a study from the Surveillance, Epidemiology, and End Results (SEER) registry data. Cancer. 2007;110(10):2191-2201.

21. Artinyan A, Hellan M, Mojica-Manosa P, et al. Improved survival with adjuvant external-beam radiation therapy in lymph node-negative pancreatic cancer: a United States population-based assessment. Cancer. 2008;112(1):34-42.

22. Yovino S, Maidment BW, Herman JM, et al. Analysis of local control in patients receiving IMRT for resected pancreatic cancers. Int J Radiat Oncol Biol Phys. 2012;83(3):916-920.

23. Ansari D, Chen BC, Dong L, Zhou MT, Andersson R. Pancreatic cancer: translational research aspects and clinical implications. World J Gastroenterol. 2012;18(13):1417-1424.

24. Fisher SB, Patel SH, Bagci P, et al. An analysis of human equilibrative nucleoside transporter-1, ribonucleoside reductase subunit M1, ribonucleoside reductase subunit $\mathrm{M} 2$, and excision repair crosscomplementing gene-1 expression in patients with resected pancreas adenocarcinoma: implications for adjuvant treatment. Cancer. 2013;119(2):445-453. 
Gastrointestinal Cancer: Targets and Therapy

Dovepress

\section{Publish your work in this journal}

Gastrointestinal Cancer: Targets and Therapy is an international, peer-reviewed, open access journal focusing on gastro-intestinal cancer research, identification of therapeutic targets and the optimal use of preventative and integrated treatment interventions to achieve improved outcomes, enhanced survival and quality of life for the cancer patient. The manuscript management system is completely online and includes a very quick and fair peer-review system. Visit http://www.dovepress.com/testimonials.php to read real quotes from published authors.

Submit your manuscript here: http://www.dovepress.com/gastro-intestinal-cancer-targets-and-therapy-journal 